282 Hansen: Über Temperatur- u. Druckbestimmung etc.

\title{
Über Temperatur- und Druckbestimmung bei Vakuumdestillationen;
}

\author{
von

\section{Christian Johannes Hansen,}

In einer erneuten Publikation ${ }^{1}$ ) hat Herr C. v. Rechenberg meine gegen seine erste Arbeit ${ }^{2}$ ) gerichtetete sachliche $\mathrm{Kritik}^{3}$ ) in persönlicher Art beantwortet, worauf ich nicht einzugehen brauche. Da indessen eine Reihe seit einiger Zeit verfolgter Versuche zur Aufklärung der diskutierten Erscheinungen vorliegt, die ich im Zusammenhang mit einer umfassenden theoretischen Untersuchung der Phänomene später veröffentlichen werde, so halte ich es immerhin für zweckmäBig, schon jetzt die Ansichten in aller Kürze darzulegen, die ich mir über die Entstehung der von Herrn v. Rechenberg zum Gegenstand seiner Theorien gemachten Temperaturabnahmen an im hohen Vakuum erzeugten Dampfsäulen auf Grund dieser $\nabla$ ersuche gebildet habe.

Herr v. Rechonberg ist der Ansicht, für die er aber den experimentellen wie den theoretischen Beweis schuldig bleibt, da $B$ diese Temperaturabnahmen durch Dampfrerluste infolge Kondensation zustande kämen; denn selbst in sehr weiten Gefäßen müsse dann noch im GefäB selbst eine Drosselung des sich äuBerst rapide entwickelnden Dampfes stattinden. ${ }^{4}$ Diese Ansicht ist prinzipiell unrichtig. Denn wenn man, wie ich es in zablreichen Fällen ausgeführt habe, seine Beobachtungen in $4 \mathrm{~cm}$ weiten zylindrischen Gefäßen anstellt, die einen vollkommenen Wärmeschutz durch mehrere konzentrische Dampfräume und einen das ganze Gefäß einschließenden Siedemantel konstanter Temperatur besitzen, so bleiben die Temperaturdifferenzen unter

1) Dies. Journ. [2] 80, 547 (1909).

Daselbst 79, 475 (1909). 's) Daselbst 80, 455 (1909).

4) Daselbst 80, 551 (1909). 
Hansen: Ūber Temperatur- u. Druckbestimmung etc. 283

allen Umständen stets von derselben GröBenordnung wie bei den Krafftschen Versuchen.

Da weiter diese Differenzen in drosselungsfreien und gegen Kondensation völlig geschützten Apparaten selbst bei $30 \mathrm{~mm}$ noch wesentliche Beträge ausmachen können, so lassen sich eindeutige Siedepunkte ohne Korrektur nach der dyna. mischen Siedemethode hier uberhaupt nicht angeben. Darin liegt der Grund, warum ich schein bar von Siedepunkten bei $0 \mathrm{~mm}$, also keinem Druck, gesprochen babe. Herr v. Rechenberg übersieht nämlich immer noch, daB ich dabei in der von $F$. Krafft eingeführten Weise stets noch die Höhe der Dampfsäule über der Thermometerkugel angebe, damit also auch den von ihm vermiBten Druck berücksichtige. $E_{s}$ ist also die Behauptung, ich gäbe Siedepunkte unter gar keinem Druck an, als unberechtigt zu bezeichnen. Ebensowenig kann es unwissenschaftlich genannt werden, wenn ich die in einem sehr hohen Vakuum vorgenommene Destillation als eine "Destillation im absoluten Vakuum" bezeichne. Dieser Ausdruck ist natürlich nur eine abgekürzte Bezeichnung der hier in besonders charakteristischer Weise vorsichgehenden Erscheinung, wobei absolut nicht gesagt wird, daB die Dämpfe der destillierenden Substanz dabei unter keinem Druck ständen, weil ja stets noch die Angabe der Dampfschicht gefordert wird. Denn wenn auch die Dämpfe in diesem Fall unter ganz merklichen Drucken von der Größenordnung des Millimeters stehen, so ist es doch nur unter gro Bem A ufwand ron Zeit und experimentellen Bilfsmitteln möglich, diese Drucke an der Stelle der Thermometerkugel zu messen und namentlich im chemischen Laboratorium völlig ausgeschlossen. Daher wäre für

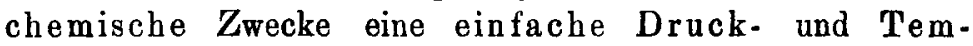
peraturangabe praktisch völlig wertlos. Denn man würde ja bei der Ausführung einer Destillation wieder in dieselbe schwierige Lage versetzt wie vorher bei der Druckbestimmung, weil man erst genau in derselben umständlichen Weise wieder einen Punkt in einer Dampfsäule ausfindig machen müBte, wo gerade wieder einmal der angegebene Druck herrschte; die einfache Druckangabe für den dampffreien Raum genügte ja wegen der höheren Drucke in der Dampfsäule nicht dazu. Es bleibt für die Praxis des chemi. 
284 Hansen: Über Temperatur- u. Druckbestimmung etc.

schen Laboratoriums aỉso nur der bisher allgemein benutzte Weg, den Druck im dampffreien Raum, der praktisch oft gleich 0 zu setzen ist, anzugeben, und zur richtigen Ergänzung des Druckes, ohne dessen Größe in $m m$ präcise angeben zu können, unter vergleichbaren Steighöhen zu arbeiten. Denn dann erhält man einen vergleichbaren Temperatur. und Druckverlauf in den Dampfsäulen, also vergleichbare Resultate, wie das praktisch ja in zahlreichen Fällen erprobt worden ist. Wie ich indessen bereits früher betont habe, ist die von Herrn v. Rechenberg vorgeschlagene Anordnung des Manometers am Siedekolben nicht geeignet, Druckdifferenzen im Kolbenhals selbst auch nur bei $15 \mathrm{~mm}$ festzustellen. Denn in den Zuleitungsröhren kondensiert sich bereits so viel Substanz, daß dadurch Überdrucke des Dampfes direkt aufgehoben werden, wovon man sich leicht durch Vergleich zweier am Kolbenhals in verschiedener Höhe angebrachter Manometer überzeugen kann. Unter diesen Umständen ist die Behauptung des Hrn. v. Rechenberg, Siedepunktsbestimmungen von F. Krafft und Mitarbeitern bei geringsten Drucken erwiesen sich jedesmal als völlig falsch, wenn man sie mit Beobachtungen anderer Forscher vergleichen könne, grundlos; denn der allerdings zu niedrige Manometerdruck ist jedesmal noch um den Druck der Dampfoäule über der Thermometerkugel zu vergrößern.

Die ganze Streitfrage kommt also einfach darauf hinäus, daB die statische und die Siedepunkts-Methode, Dampfspannungsmessungen anzustellen, nicht ohne weiteres dieselben Resultate liefern, weil bei einer Siedepunktsbestimmung hochmolekularer Körper unter geringen Drucken noch der Eigendruck des strömenden Dampfes zu berücksichtigen ist, der bei der statischen Methode fehlt. Setzt man freilich diesen Druck in Rechnung, was sich aber im chemischen Laboratorium nicht ausführen läßt, dann werden natürlich die Resultate identisch, wie es die Theorie erfordert. Es bleibt also für das chemische Laboratorium der einzige Weg: Angabe des Druckes im dampfreien Raum und als Ergänzung Angabe der Höhe der Dampfsäule.

Um so verwunderlicher erscheint es nun freilich, dab Herr v. Rechenberg, der sich übrigens zur Unterstützung 
Hansen: Über Temperatur- u. Druckbestimmung etc. 285

seiner Ansichten auf seine über 20-jährige Erfahrung im Destillationswesen als Direktor in der Fabrik von Schimmel \& Co. in Miltitz bei Leipzig beruft, an. dauernd die praktisch zu ermittelnden Siedepunkte normal über $300^{\circ}$ siedender Stoffe ganz einfach mit den nach der statischen Metbode erhaltenen Spannkraftsmessungen identifiziert, obwohl er selbst auf S. 554 ausdrücklich erklärt, er hielte die dynamische Methode bei so hochmolekularen Substanzen über. haupt nicht für geeignet!

Da im übrigen die Drucke in den Dampfeäulen von der Größenordnung des Millimeters sind, so ist klar, da $B$ ein Evakuieren auf weniger als etwa $0,01 \mathrm{~mm}$ diese Erscheinungen nicht mehr wesentlich merkbar beeinfluBt. Dieser Grenzdruck im dampffreien Raum wird daher als für diesen Fall nicbt mehr merklich direkt mit $0 \mathrm{~mm}$ bezeichnet ${ }^{1}$ ), während die eigentliche praktisch in Millimetern nicht ohne weiteres zu ermittelnde Druckangabe in der Angabe der Höhe der Dampfschicht liegt.

Im Prinzip ist sodann die Ansicht des Herrn vo Rechenberg, die Druckabnahmen kämen durch Drosselung infolge auBerordentlich großer Dampfgeschwindigkeiten zustande, nicht haltbar. Sie baben eine ganz andere Ursache. Es ist nämlich bei der Entwicklung eines Dampfes aus einer ursprünglich unter minimalem Druck stehenden Flüssigkeit zur Erlangung einer gewissen Strömungsgeschwindigkeit desselben stets ein Druckgefälle erforderlich, selbst wenn das Getäß bei mäßiger Geschwindigkeit bequem den Dampf ohne Drosselung aufnehmen kann. Zwar macht bei einem gesättigten Dampf von Atmosphärendruck ein solches Gefälle für die Temperaturbestimmung bei der geringen Siedepunktsänderung mit dem Druck nur wenig aus, soda $B$ man gewöhnlich sogar einige Millimeter Druckdifferenz vernachlässigen kann. Bei einer unter minimalem Druck im dampffreien Raum vorgenommenen Destillation ist die Sachlage dagegen eine wesentlich andere. Denn hier entspricht $1 \mathrm{~mm}$ Druckänderung eine bedeutende Änderung der Temperatur; vor allem aber wächst, wenn man z. B. von $0,001 \mathrm{~mm}$ auf $1 \mathrm{~mm}$ heraufgeht, gleichzeitig die

1) Eine sich wohl hierauf beziehende Bemerkung von Stock und Heynemann [Ber. 42, 4088 (1909)] berubt also auf einem kleinen MiBverständnis. 
Dampfdichte auf den 1000-fachen Betrag, so daB man dann sofort brauchbare Bedingungen für eine praktische Destillation erhält, ohne dabei mit v. Rechenberg zu groBe Dampfgeschwindigkeiten voraussetzen zu müssen. Berücksichtigt man nun nocb den Umstand, daB bei einer freien Diffusion eines hochmolekularen Dampfes in ein Gas von gleicher Temperatur und gleichem Druck von etwa 0,001 mm die im Anfang in der Zeiteinheit durch Diffusion bei konstantem Gesamtdruck durch einen bestimmten Querschnitt fortgeschaffte Menge Substanz nur minimal ist, so ist klar, daB man - und damit hat man es hier zu tun - ein Gesamtdruckgefälle bekommt, sowie man durch Energiezufuhr zu einer Flüssigkeit unter obigem Anfangszustand mehr Dampf erzeugt, als eine freie Diffusion bei konstantem Gesamtdruck fortschaffen könnte. In Übereinstimmung damit, daß Druckdifferenzen bei ganz geringen Drucken zum Ausgleich eine sehr merkliche Zeit brauchen, erklärt es sich dann, daB in völlig drosselungsfreien Gefäßen über einer im hohen Anfangsvakuum angeheizten Substanz sich je nach der Stärke der Heizung eine Dampfschicht bildet, in der ein erhebliches Druckgefälle stattfindet. Das Experiment zeigt, daB dies Gefälle mehrere Millimeter betragen kann und unter den gewöhnlichen Bedingungen beträgt; diesem jetzt relativ hohen Druck hat es dann der Dampf zugleich zu verdanken, wenn er praktisch brauchbare Dichte erhält. Dann genügen aber auch die von mir benutzten weiten Gefäße von vornherein, die entwickelte Dampfmenge ohne Drosselung aufzunehmen, so daß die Destillation bei mäßiger Dampfgeschwindigkeit und obne zumal bei dünnen Dämpfen in weiten Gefäßen nicht sehr merkliche Reibung vorsichgeht.

Damit sind die Ansichten des Herrn v. Rechenberg, der von vornherein mit diesen selbst ohne Drosse. lung an und für sich unmöglichen großen, sehr dünnen Dämpfen entsprechenden Dampfgeschwindigkeiten rechnet, erledigt. Denn er sucht die Erscheinungen ohne irgendwelche Berücksichtigung dieser typischen Diffusionserscheinung infolgedessen nur durch Reibung und diese verstärkende $K$ ondensationsverluste zu erklären, bei deren AusschluB sich das Problem aber, wie das 
Hansen: Uber Temperatur- 1. Druckbestimmung etc. 287

Experiment gezeigt hat, genau so stellt. Er geht somit auf das Prinzipielle der ganzen Frage uberhaupt nicht mit einem Worte ein,

Diese prinzipielle Erklärung der Druckabnahmen schließt natürlich nicht aus, daß eine Drosselung des Dampfes sowie Kondensationsverluste diese Differenzen verstärken werden. Wie aber Herr v. Rechenberg dazu kommt, bei dem Krafft-Lehmannschen ${ }^{1}$ ) Versuch eine stärkere Drosselung anzunehmen, ist mir nicht klar; denn daun müßten doch gerade die Druckabnabmen sowie die entsprechenden Temperaturdifferenzen sich vergrößern. Faktisch werden sie aber kleiner!

Aus obiger Auffassung folgt sodann, daB die Temperaturabnahmen in den Dampfsäulen in Übereinstimmung mit meinen Versuchen zwar von der AuBentemperatur ihrer absoluten Höhe nach weitgehend beeinfußt werden können, andererseits aber auch selbst durch Überhitzen nicht verschwinden werden. Infolgedessen wird durch den von mir bereits gerügten Vorschlag des Herrn v. Rechenberg zunächst das eigentlich beabsichtigte, nämlich Erzielung eines einheitlichen Druckes und einheitlicher Temperatur im Gefäß, gar nicht erreicht; vielmehr bleiben die Differenzen immer noch bestehen. Sodann wird aber dadurch einer geradezu völligen Willkür in der Temperaturmessung Tür und 'Tor geöffnet, da gerade für geringste Drucke das völlige Eintauchen des Siedegefäßes in das Bad das Schlimmste ist, was man tun kann; auf diese Weise gewonnene Zahlen sind dann überhaupt gewöhnlich völlig unbrauchbar, wie der Versuch im Gegensatz zu der Schätzung des Herrn v. Rechenberg mir oft genug gezeigt hat.

Man erkennt jetzt auch, daß das von Herm v. Rechenberg zur Unterstützung seiver Kondensationstheorie gewählte Beispiel von Ben. zol- und Quecksilberdampf von $80^{\circ}$ und 755 bzw. 0,09 mm Druek unzulässig ist, da ein entsprechender Versuch mit destillierendem Quecksilber in der Form einer Destillation durch willkürliche Heizung gar nicht realisiert werden kann. Denn sowie, und das geschieht eben stets, die Temperatur des Quecksilbers über $80^{\circ}$ steigt, erbält man auch Dampf von höberem Druck und entsprechend höherer Dichte. Dementsprechend liegen die Krafftschen Drucke und Temperaturen einem wirklich ausgeführten Experiment gemä $B$ bei mehreren Millimetern Druck und $151^{\circ}$ bis $178^{\circ}$ !

Ich kann mir zum Schluß nicht versagen darauf hinzuweisen, da $B$ man nicht, wie es Hr. v. Rechenberg unternommen hat, die Druckgefalle einfach sus dem aus Länge der Dampfschicht und der dem im dampffreien Raum beobachteten Druck entaprechenden Dampfdichte ermittelten Dampfgewicht berechnen darf; denn man hat es ja nicht mit einem statischen Phänomen, sondern mit strömendem Dampf zu tun, obwohl das eigentlich als selhstverständlich vorausgesetzt werden sollte!

1) Ber. 38, 246 (1905). 
288 Hansen: Ober Temperatur- u. Druckbestimmung etc.

Auf die vielen weiteren Momente, die bei den vorliegenden Fragen als ausschlaggebend in Betracht kommen und deren es naturgemäB noch eine ganze Anzahl wichtiger gibt, kann ich hier nicht eingehen. Im übrigen behalte ich mir ausdrücklich vor, die meine Auffassung beweisenden Experimente erst später im Zusammenhang mit einer theoretischen Behandlung der Erscheinungen bei einer Destillation unter ganz kleinen Drucken zu bringen, wozu es mir jetzt an Zeit fehlt. Bei dieser Gelegenheit werde ich dann auf manches Fehlende ausführlich eingehen, was der beschränkte Raum mir hier nicht gestattet.

Leipzig, Chemisches Laboratorium der Universität. 